\title{
Three-dimensional Surface Charge Reconstructions of Surface Plasmon Modes of Silver Right Bipyramids
}

\author{
Sean M. Collins ${ }^{1}$, Martial Duchamp ${ }^{2}$, Emilie Ringe ${ }^{3}$, Zineb Saghi ${ }^{1}$, and Paul A. Midgley ${ }^{1}$. \\ 1. Department of Materials Science and Metallurgy, University of Cambridge, Cambridge, UK. \\ 2. Ernst Ruska-Centre, Forschungszentrum Jülich, Jülich, Germany. \\ 3. Department of Materials Science and Nanoengineering, Rice University, Houston, USA.
}

Plasmonic noble metal nanoparticles enable nanoscale confinement of electromagnetic fields for the development of chemical and biological sensing, photonic devices, and light trapping technologies. Scanning transmission electron microscopy and electron energy loss spectroscopy (STEM-EELS) has emerged as a key tool in experimentally observing the near field plasmonic modes of a wide variety of nanoparticle shapes and sizes [1]. When nanoparticle are at least approximately thin and invariant along the direction of the electron beam, the EELS signal can be related to a photonic localized density of states (LDOS) [2]. This LDOS, however, does not describe the three-dimensional fields or potentials surrounding the plasmonic nanoparticle [3], particularly in prismatic nanocrystals common in noble metal nanoparticle synthesis. Qualitative measurement of the three-dimensional EELS response has been demonstrated for a silver nanocube [4]. An alternative approach to reconstruct the surface charges corresponding to an eigenmode decomposition of the EELS response has been proposed theoretically [5], but has not been demonstrated experimentally to date.

Here, we present surface charge reconstructions of the principal modes of silver right bipyramids. Experimental tilt-series STEM-EELS was acquired on the PICO microscope, a modified FEI Titan operated at $300 \mathrm{kV}$ and equipped with a monochromator and a Quantum GIF for fast spectroscopy acquisition. Chemically synthesized silver right bipyramids with side length of approximately $50 \mathrm{~nm}$ were drop-cast onto molybdenum trioxide $\left(\mathrm{MoO}_{3}\right)$ crystals dispersed on lacey carbon TEM grids. A straight-forward reconstruction of surface charge uses non-penetrating trajectories only [5], and so a partial $\mathrm{MoO}_{3}$ crystal substrate with a bipyramid resting against a crystal side in cross-section was selected for tilt-series STEM-EELS measurements. An annular dark field (ADF) tilt series was acquired concurrently with STEM-EELS for structural tomography of the particle morphology. ADF images were acquired in $3^{\circ}$ increments from $68^{\circ}$ to $-67^{\circ}$. STEM-EELS maps were acquired at $0^{\circ}$ and every $9^{\circ}$ from $65^{\circ}$ to $38^{\circ}$, limited by carbon contamination. The STEM-EELS data was processed by nonnegative matrix factorization (NMF) to separate modal contributions. Compressed sensing electron tomography (CS-ET) [6] was performed on the ADF data, the bipyramid morphology was extracted from the tomogram by threshold-based segmentation, and a surface mesh was generated for use in boundary element method (BEM) [7] simulations and surface charge reconstructions.

The proposed reconstruction technique [5] uses conjugate gradient methods to minimize a cost function of the least-squares norm of the experimental data and a forward-calculated EELS response from a surface charge model. Given the prior knowledge of the particle morphology determined from CS-ET, the initial input surface charge distribution for cost function minimization was determined from BEM eigenmode calculations. To further constrain the cost function optimization algorithm to handle experimental noise and artifacts, an additional soft thresholding regularization penalty was used to identify solutions to the surface charge optimization that exhibited a small number of surface elements with high intensity, consistent with the experimental data and the highly localized surface charge 
densities typical of corner modes.

Figure 1 presents surface charge reconstructions for two of the four principal corner modes observed experimentally. In the tilt series, two overlapping modes at $2.7 \mathrm{eV}$ were not separated by NMF. However, two-dimensional maps of additional silver bipyramids revealed two separate modes in this spectral window. As determined from BEM simulations, the corner modes in an isolated right bipyramid comprise three dipole $((x, y)$ and $z)$ and one quadrupole $\left(z^{2}\right)$ excitation, showing good agreement with the experimentally recovered modes. Symmetry-breaking by the substrate separates the degenerate $(x, y)$ modes in the isolated bipyramid into modes at $1.3 \mathrm{eV}$ and $1.8 \mathrm{eV}$. Significant surface charges on the substrate are observed in part due to artifacts in the experimental EELS maps exhibiting signal along the substrate-vacuum boundary. The method may be extended to higher energy modes localized along the edges and faces of the bipyramid, though these modes present additional challenges due to a high degree of multipolar mode overlap. The surface charge reconstructions presented here represent an excitationindependent response and can be used to predict the response to general illuminating fields with near or far field sources, offering a direct and quantitative assessment of the optical response of plasmonic particles from EELS [8].

\section{References:}

[1] FJ García de Abajo, Reviews of Modern Physics 82 (2010), p. 209.

[2] FJ García de Abajo and M Kociak, Physical Review Letters 100 (2008), p. 106804.

[3] U Hohenester, H Ditlbacher and JR Krenn, Physical Review Letters 103 (2009), p.106801.

[4] O Nicoletti et al, Nature 502 (2013), p. 80.

[5] A Hörl, A Trügler and U Hohenester, Physical Review Letters 111 (2013), p. 076801.

[6] R Leary et al, Ultramicroscopy 131 (2013), p. 70.

[7] U Hohenester, Computer Physics Communications 185 (2014), p. 1177.

[8] SM Collins acknowledges support of a Gates Cambridge Scholarship. The authors acknowledge funding from the European Research Council under the European Union's Seventh Framework Program (Grant No. FP7/2007-2013)/ERC Grant Agreement No. 291522-3DIMAGE and from the European Union's Seventh Framework Program under a contract for an Integrated Infrastructure Initiative (Reference No. 312483-ESTEEM2).
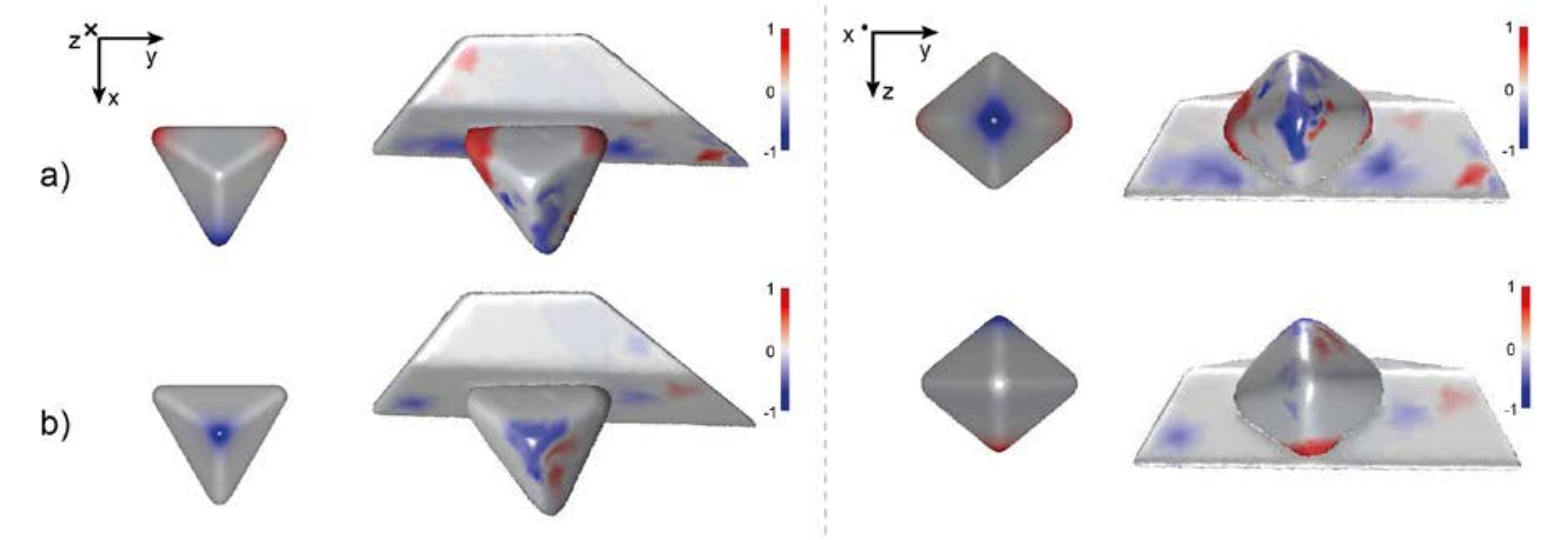

Figure 1. Simulated and experimentally recovered surface charge distributions for (a) $x$ and (b) $z$ dipolar corner modes of a silver right bipyramid (each shown at two orientations). Surface charges are shown on a normalized color scale. Axes (top left) indicate the relative coordinates of each orientation. 